\title{
Behavior of single nanoparticle/homopolymer chain in ordered structures of diblock copolymers
}

\author{
Qiang Wang, ${ }^{\text {a) }}$ Paul F. Nealey, and Juan J. de Pablo ${ }^{\text {b) }}$ \\ Department of Chemical Engineering, University of Wisconsin-Madison, Madison, Wisconsin 53706-1691
}

(Received 23 September 2002; accepted 26 March 2003)

\begin{abstract}
We have performed Monte Carlo simulations to study the behavior of a single nanoparticle and a single homopolymer chain in ordered structures of diblock copolymers. Our results show that the microphase-separated copolymers can be used to position nanoparticles or homopolymers according to their interactions with the two blocks. This is consistent with recent experimental observations. We also observe that, while short neutral homopolymers segregate at the A-B interfaces, longer neutral homopolymers exhibit an apparent preference for the cylinders over the matrix, mainly due to geometrical considerations associated with the curvature of A-B interfaces. (C) 2003 American Institute of Physics. [DOI: 10.1063/1.1575207]
\end{abstract}

\section{INTRODUCTION}

Due to their ability to microphase-separate into ordered structures on the length scale of tens of nanometers, block copolymers are receiving increased attention for potential applications in nanofabrication. ${ }^{1}$ For example, ordered copolymer structures can be used as templates to guide the spatial arrangement of nanoparticles ${ }^{2}$ to manufacture selective membranes, next-generation catalysts, photonic crystals, etc. ${ }^{3}$ For these materials, a desirable morphology is attained by controlling the copolymer composition, the volume fraction of the particles, the interaction between the particles and the blocks, etc.

To the best of our knowledge, only a few theoretical studies have been reported on nanoparticle/copolymer systems. Sevink et al. used a dynamic mean-field density functional theory to study the phase separation of symmetric diblock copolymers in the presence of immobile filler particles. ${ }^{4}$ Ginzburg et al. developed a hybrid mesoscopic model that combines cell dynamic simulations for the copolymers with Langevin dynamics for the particles to study the phase separation of nanoparticle/symmetric diblock copolymer systems. ${ }^{5,6}$ In these numerical calculations, molecular details about chain conformation were lost due to the mesoscopic nature of the models. Huh et al. used lattice Monte Carlo simulations and the strong-stretching theory to study the phase behavior of cubic nanoparticle/diblock copolymer systems. ${ }^{7}$ Thompson et al. combined a selfconsistent mean-field theory for the copolymers with a density functional theory for the particles, and performed 2D calculations to explore the phase behavior of spherical nanoparticle/diblock copolymer systems. ${ }^{8-10}$ Although their work did not assume a priori knowledge of the system's morphology, the two-dimensional aspects of the work limits the applicability of its predictions.

\footnotetext{
a) Current address: Department of Chemical Engineering and the Materials Research Laboratory, University of California-Santa Barbara, Santa Barbara, California 93106.

b) Author to whom correspondence should be addressed.
}

In this work, we report lattice Monte Carlo simulations for systems of a single nanoparticle in lamellae of symmetric diblock copolymers. For the microphase-separated block copolymers, the addition of nanoparticles can change the characteristic dimensions, or even the morphology, of the ordered copolymer structures. This can be problematic in constantvolume simulations, where it is difficult to avoid the influence of the periodic boundary conditions on the ordered structures. ${ }^{11,12}$ In contrast to previous work, this issue is minimized in our single-particle simulations. Although the problem at hand is also simplified because the particleparticle interactions are not considered, our simulations are still able to provide useful insights into the systems of interest.

We also report lattice Monte Carlo simulations for systems of a single homopolymer chain in cylinders of asymmetric diblock copolymers. Although Monte Carlo simulations on the blends of block copolymers with homopolymers have been reported in the literature, ${ }^{13}$ they have mainly focused on the behavior of copolymers at homopolymer interfaces and on the phase behavior of the system; singlehomopolymer simulations have not been reported before. Our work is motivated by recent experiments of Jeong et al., where poly(methylmethacrylate) (denoted by PMMA) homopolymers were added into asymmetric diblock copolymers polystyrene- $b$-PMMA (where PMMA blocks form cylinders of about $22 \mathrm{~nm}$ in diameter), and pores of about $6 \mathrm{~nm}$ in diameter were obtained after removing the homopolymers. ${ }^{14}$ This size scale is generally beyond the bounds available to block copolymers alone, and extends the potential applications of block copolymer systems. As shown later, our results are consistent with experimental observations.

\section{MODEL}

Our Monte Carlo simulations are performed in an expanded grand-canonical ensemble in the framework of a cubic lattice. Detailed descriptions of our model can be found in Refs. 11, 12, and 15. Simulation boxes of size $L_{x} \times L_{y}$ 
$\times L_{z}$ are employed, and periodic boundary conditions are applied in all three directions. We only consider repulsions between nonbonded nearest-neighbor $\mathrm{A}-\mathrm{B}$ pairs separated by one lattice unit $\left(\epsilon_{\mathrm{A}-\mathrm{B}}>0\right)$, and set $\epsilon_{\mathrm{A}-\mathrm{A}}=\epsilon_{\mathrm{B}-\mathrm{B}}=0$. Any interactions involving vacancies (unoccupied lattice sites) are also set to zero.

\section{A. Single nanoparticle in lamellae of symmetric diblock copolymers}

In this case, cubic boxes of size $28^{3}$ or $42^{3}$ are employed. The symmetric diblock copolymers have a chain length of 24. The reduced temperature is set to $T^{*}$ $\equiv k_{B} T / \epsilon_{\mathrm{A}-\mathrm{B}}=1.2$ (where $k_{B}$ is Boltzmann's constant and $T$ is the absolute temperature), and the reduced chemical potential to $\mu^{*} \equiv \mu /\left(k_{B} T\right)=43.4$ (where $\mu$ is the chemical potential of the copolymer chain). Under these conditions, the symmetric diblock copolymers form lamellar structures of period $L=14$ along one axis of the box (denoted by $z$ ), and the copolymer segmental density (fraction of lattice sites occupied by copolymer segments) $\bar{\phi}_{\mathrm{C}}$ fluctuates around 0.8 during the simulation. ${ }^{15}$

A single particle (denoted by $\mathrm{P}$ ) is fixed at the center of the box. Two different shapes of the particle are considered: a cubic particle consisting of $l^{3}$ lattice sites, and a spherical particle consisting of all the lattice sites whose distance from the center of the box is smaller than or equal to $d / 2$. Because of the lattice structure, the latter is actually a quasispherical particle. The lattice sites occupied by the particle cannot be occupied by copolymer segments. We set $\epsilon_{\mathrm{A}-\mathrm{P}}=0$ and $\epsilon_{\mathrm{B}-\mathrm{P}}$ $\geqslant 0$, and define $\alpha_{\mathrm{P}} \equiv \epsilon_{\mathrm{B}-\mathrm{P}} / \epsilon_{\mathrm{A}-\mathrm{B}}$. Therefore, $\alpha_{\mathrm{P}}=0$ corresponds to a neutral particle, and $\alpha_{\mathrm{P}}=2$ corresponds to an A-like particle.

Metropolis-like acceptance criteria are used in these simulations. One Monte Carlo step (MCS) consists of 0.8 $\times L_{x} \times L_{y} \times L_{z}$ trials of reptation, local, and growing/ shrinking moves of copolymer chains, each of which occurs with the same probability. After equilibration, simulations are run for at least $1 \times 10^{6} \mathrm{MCS}$ and data are collected every $10 \mathrm{MCS}$.

\section{B. Single homopolymer chain in cylinders of asymmetric diblock copolymers}

In this case, a rectangular box of size $54 \times 52 \times 60$ is employed. The asymmetric diblock copolymers have a chain length of 36 and a composition (the volume fraction of the A blocks in the copolymers) of $1 / 4$. The temperature and density are set to $T^{*}=1.5$ and $\bar{\phi}_{\mathrm{C}}=0.693$. Under these conditions, the asymmetric diblock copolymers form hexagonally packed cylinders of the A blocks in the matrix of the B blocks. These cylinders are oriented along the $z$ direction, with an intercylinder distance of 17.3 along the $y$ direction. $^{12,16}$

A single homopolymer chain, denoted by $\mathrm{H}$, of varying chain length $N$ is introduced into the ordered cylindrical structures via expanded grand-canonical Monte Carlo moves. ${ }^{11}$ In each of these growing/shrinking moves, two segments are appended to or removed from each end of the homopolymer chain. Configurational bias is used to facilitate these moves. ${ }^{11}$ In addition, local (kink-jump and crankshaft) moves and reptation moves are employed for both the copolymers and the homopolymer to change their chain configuration. We set $\epsilon_{\mathrm{H}-\mathrm{H}}=0, \epsilon_{\mathrm{A}-\mathrm{H}} \geqslant 0, \epsilon_{\mathrm{B}-\mathrm{H}} \geqslant 0, \epsilon_{\mathrm{A}-\mathrm{H}} \epsilon_{\mathrm{B}-\mathrm{H}}$ $=0$, and define $\alpha_{\mathrm{H}} \equiv\left(\epsilon_{\mathrm{B}-\mathrm{H}}-\epsilon_{\mathrm{A}-\mathrm{H}}\right) / \epsilon_{\mathrm{A}-\mathrm{B}}$. Therefore, $\alpha_{\mathrm{H}}$ $=0$ corresponds to a neutral homopolymer (denoted by $\left.\mathrm{H}^{\mathrm{N}}\right)$, $\alpha_{\mathrm{H}}=1$ corresponds to an A-like homopolymer (denoted by $\mathrm{H}^{\mathrm{A}}$ ), and $\alpha_{\mathrm{H}}=-2$ corresponds to a B-like homopolymer (denoted by $\mathrm{H}^{\mathrm{B}+}$ ).

Metropolis-like acceptance criteria are used in these simulations. One MCS consists of $0.7 \times L_{x} \times L_{y} \times L_{z}$ trials of reptation, local, and growing/shrinking moves, occurring with probability $0.45,0.45$, and 0.1 , respectively. The copolymers and the homopolymer are chosen with the same probability (0.5) for reptation and local trial moves, while the growing/shrinking trial moves are only employed for the homopolymer. The configuration of the single homopolymer chain is therefore changed much more frequently than that of the copolymers. We start from a well-equilibrated system configuration of the copolymers, and simulations are run for $1 \times 10^{6} \mathrm{MCS}$ while data are collected every $1 \mathrm{MCS}$.

\section{RESULTS AND DISCUSSION}

\section{A. Single nanoparticle in lamellae of symmetric diblock copolymers}

In order to employ ordered structures of block copolymers to position nanoparticles, the particle size has to be comparable to or smaller than the domain size of the copolymers ( $L / 2$ in the case of our lamellae). Two different particle sizes are considered in this work: "small" particles of $l=6$ or $d=6$ (where the box size of $28^{3}$ is used), and "large" particles of $l=10$ or $d=12.4$ (where the box size of $42^{3}$ is used). In the latter case, the spherical particle has about the same volume as the cubic one.

The left section of Fig. 1 shows the position of the four lamellar interfaces in the $z$ direction during the simulation for the case of "small" particles. The interfacial positions in each collected system configuration are defined by $\phi_{\mathrm{A}}(z)$ $-\phi_{\mathrm{B}}(z)=0$, where $\phi_{\mathrm{A}}(z)$, for example, is the fraction of lattice sites occupied by A segments in the $x-y$ plane at a given $z$. Periodic boundary conditions are used when calculating the interfacial positions. Each solid horizontal line (in black) represents the average position of the corresponding interface after the system is equilibrated. The two dashed lines mark the position of the particle in the $z$ direction. The right section of Fig. 1 shows one quarter of the final system configuration $\left(1 \leqslant x \leqslant L_{x} / 2\right.$ and $\left.1 \leqslant y \leqslant L_{y} / 2\right)$, where the A segments are shown as pink, the B segments are blue, and the particle is located at the corners closest to the reader.

Figure 1(a) shows that, as expected, the A-like spherical particle is preferentially located in an A-rich domain, although at the beginning of the simulation it is positioned at an A-B interface. A similar result is observed for the A-like cubic particle of $l=6$.

From Fig. 1(b) it is seen that, during the simulation, the neutral cubic particle moves (relative to the lamellae) from a B-rich domain to an A-B interface and then fluctuates around the interface. A similar result is observed for the neu- 

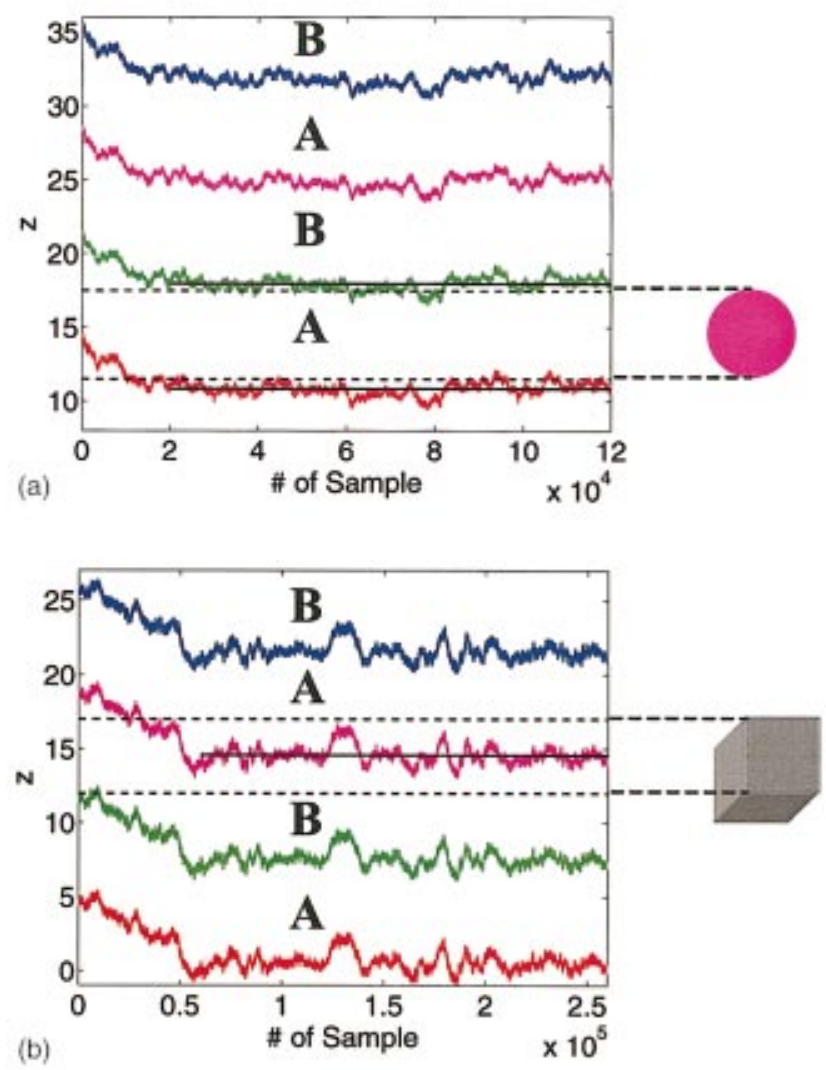

tral spherical particle of $d=6$. This clearly shows that "small" neutral particles are preferentially located at the A-B interfaces of the lamellae. Consistent with the segregation of neutral good solvent molecules at $\mathrm{A}-\mathrm{B}$ interfaces, ${ }^{11,15,17}$ this screens the repulsion between $\mathrm{A}$ and $\mathrm{B}$ segments, and therefore decreases the energy of the system.

One thing to note in Fig. 1 is that, although the particle size is close to $L / 2$, the lamellar structure is not visibly disturbed. We therefore expect that smaller particles would exhibit the same behavior. This is also true for "large" neutral particles, as shown in Fig. 2(a) for the case of a spherical particle.

In contrast, Fig. 2(b) shows that the "large" A-like cubic particle does disturb the lamellar structure; the A segments surround the particle due to energetic interactions, and perforate the middle B-rich layer. The center of an A-like particle is not always located in a B-rich layer [as shown in Fig. 2(b)]. In the case of the "large" A-like spherical particle, the center of the particle is located in an A-rich layer during our simulation, and the two adjacent B-rich layers are disturbed by the A segments surrounding the particle. As discussed below, we attribute this difference to the large box size used in these simulations (and not the different particle shapes).

As pointed out in our previous work, bulk lamellae (without the particle) also move during the course of a simulation run, due to the translational invariance of the system. ${ }^{15}$ Because the lamellar interfaces move cooperatively (as shown in Figs. 1 and 2), the motion of lamellae becomes smaller for larger simulation boxes. Since the particle is fixed in this work, in order to distinguish the motion of lamellae

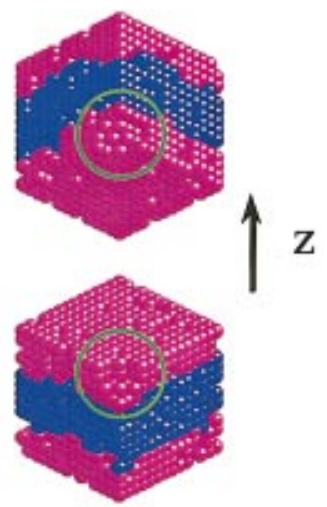

FIG. 1. (Color) The results for a "small" particle (in a simulation box of $28^{3}$ ): (a) An A-like spherical particle of $d=6$; (b) A neutral cubic particle of $l=6$. On the left shows the position of the four lamellar interfaces in the $z$ direction during the simulation, where each solid horizontal line (in black) represents the average position of the corresponding interface after the system is equilibrated, and the two dashed lines mark the position of the particle in the $z$ direction. On the right shows one quarter of the final system configuration $\left(1 \leqslant x \leqslant L_{x} / 2\right.$ and $1 \leqslant y$ $\leqslant L_{y} / 2$ ), where the A segments are shown as pink, the B segments are blue, and the particle is located at the corners closest to the reader. caused by the preferential location of the particle from that caused by the translational invariance, we compare the second moment of the lamellar interfacial position about its average position (after equilibration, denoted by $\delta$ ) with and without the particle; the results are shown in Table I. We see that, in the case of box size of $28^{3}, \delta$ is largely reduced by the particle, i.e., the lamellae are "anchored" by the preferential location of the "small" particle. In the case of box size of $42^{3}$, however, the values of $\delta$ with and without the "large" particle are comparable; our simulations are therefore unable to reveal the preferential location of the "large" particles.

\section{B. Single homopolymer chain in cylindrical structures of asymmetric diblock copolymers}

In our simulations, we consider homopolymer chain lengths in the range $0 \leqslant N \leqslant 120$. We choose the reduced chemical potential of the homopolymer $\mu_{\mathrm{H}}^{*} \equiv \mu_{\mathrm{H}} /\left(k_{B} T\right)$, where $\mu_{\mathrm{H}}$ is the chemical potential of the homopolymer chain of $N=120$, such that homopolymers of length $4 \leqslant N$ $\leqslant 120$ are sampled nearly evenly. The results are shown in Fig. 3, where $P(N)$ is the fraction of collected samples having a homopolymer of length $N$.

The first graph of Fig. 4 shows the 2D order parameter profile $O(x, y) \equiv\left\langle\phi_{\mathrm{A}}(x, y)-\phi_{\mathrm{B}}(x, y)\right\rangle$ of the copolymers in an unit cell of $27 \times 17 \frac{1}{3}$ of the hexagonal packing of the cylinders, where $\phi_{\mathrm{A}}(x, y)$, for example, is the fraction of lattice sites occupied by A segments at a given $(x, y)$ in each collected sample, and \langle\rangle represents the average over all col- 

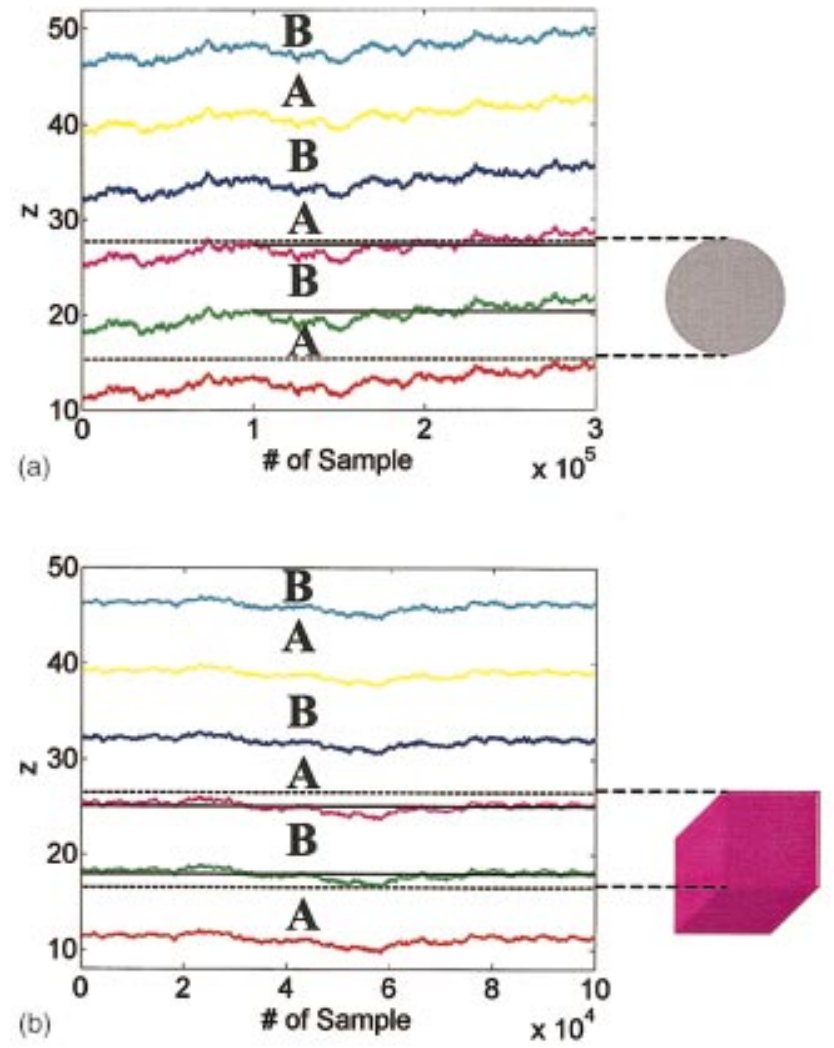

lected samples, and then over the six unit cells contained in the simulation box. Similar to the aforementioned motion of bulk lamellae, the cylinders also move in the $x-y$ plane during the course of a simulation run, due to the periodic boundary conditions. Therefore, when averaging over collected samples, we deduct this motion in a similar way to that used in previous work, ${ }^{15}$ i.e., the $2 \mathrm{D}$ order parameter profiles are aligned according to the average (over the 12 cylinders contained in the box) displacement of the cylinder's center-ofmass in the $x-y$ plane. ${ }^{18}$

The rest of Fig. 4 shows the $2 \mathrm{D}$ segmental distribution profiles $D(x, y ; N) \equiv\left(L_{x} L_{y} / N\right)\left\langle\phi_{\mathrm{H}}(x, y)\right\rangle_{N}$ of the A-like homopolymers $\mathrm{H}^{\mathrm{A}}$ of different $N$, where the prefactor $L_{x} L_{y} / N$ is used to rescale the profile such that the uniform distribution of the homopolymer segments in the system corresponds

TABLE I. Comparison of the second moment of the lamellar interfacial position about its average position (after equilibration).

\begin{tabular}{|c|c|c|c|}
\hline Box size & Particle size & $\alpha_{\mathrm{P}}$ & $\delta$ \\
\hline \multirow[t]{3}{*}{$28^{3}$} & No particle & $\cdots$ & 1.7 \\
\hline & $l=6$ & $\begin{array}{l}0 \\
2\end{array}$ & $\begin{array}{l}0.6 \\
0.4\end{array}$ \\
\hline & $d=6$ & $\begin{array}{l}0 \\
2\end{array}$ & $\begin{array}{l}0.7 \\
0.5\end{array}$ \\
\hline \multirow[t]{3}{*}{$42^{3}$} & No particle & $\ldots$ & 0.7 \\
\hline & $l=10$ & $\begin{array}{l}0 \\
2\end{array}$ & $\begin{array}{l}0.6 \\
0.5\end{array}$ \\
\hline & $d=12.4$ & $\begin{array}{l}0 \\
2\end{array}$ & $\begin{array}{l}0.9 \\
0.4\end{array}$ \\
\hline
\end{tabular}

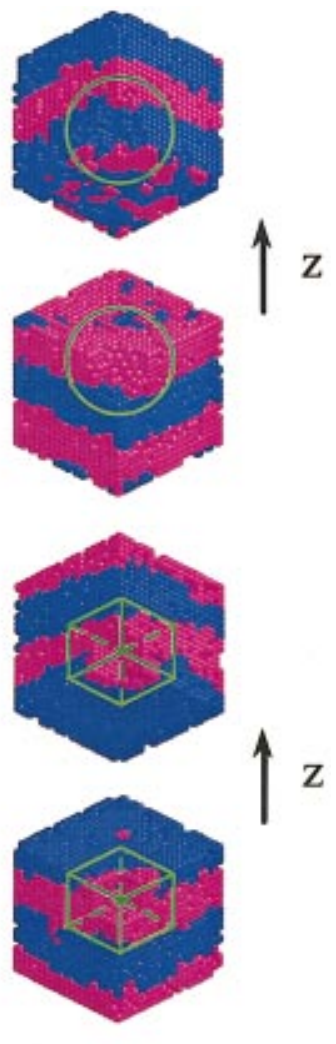

FIG. 2. (Color) The results for a "large" particle (in a simulation box of $42^{3}$ ): (a) A neutral spherical particle of $d=12.4$; (b) An A-like cubic particle of $l=10$. Refer to Fig. 1 for more explanation. to $D(x, y ; N)=1$. The notation \langle\rangle$_{N}$ here is similar to \langle\rangle , except that the average is taken only over collected samples having a homopolymer of length $N$, and the motion of cylinders is deducted as explained above. Figure 4 shows that $\mathrm{H}^{\mathrm{A}}$ is strongly localized at the center of the cylinders due to its energetic interactions; this is consistent with experimental observations. ${ }^{14}$ We also see that this localization becomes stronger as $N$ increases.

Similarly, the B-like homopolymers $\mathrm{H}^{\mathrm{B}+}$ are localized in the matrix, as shown in Fig. 5. Since the volume of the matrix is about as three times as that of cylinders, the maximum value of $D(x, y ; N)$ for $\mathrm{H}^{\mathrm{B}+}$ is much smaller than that for $\mathrm{H}^{\mathrm{A}}$.

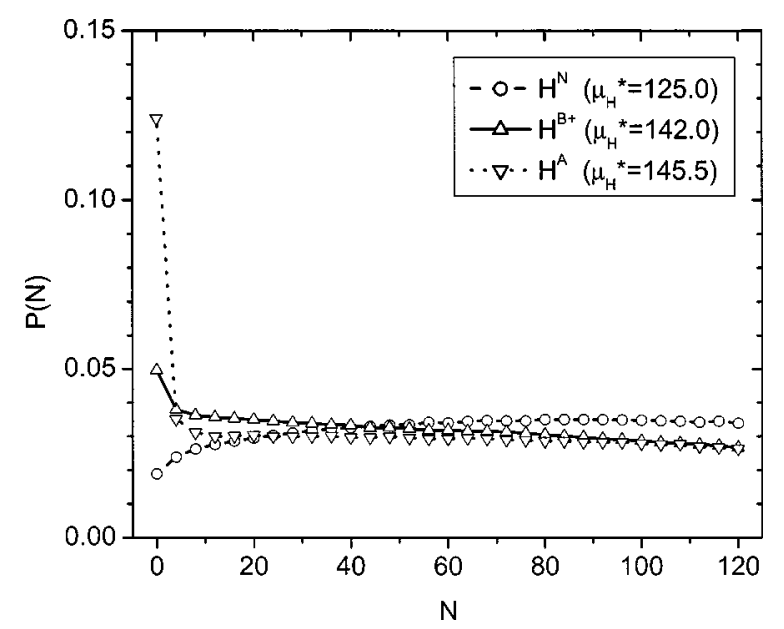

FIG. 3. Fraction of collected samples $P(N)$ having a homopolymer of length $N$. The chemical potential used for the homopolymer is shown in the legend for each case. 

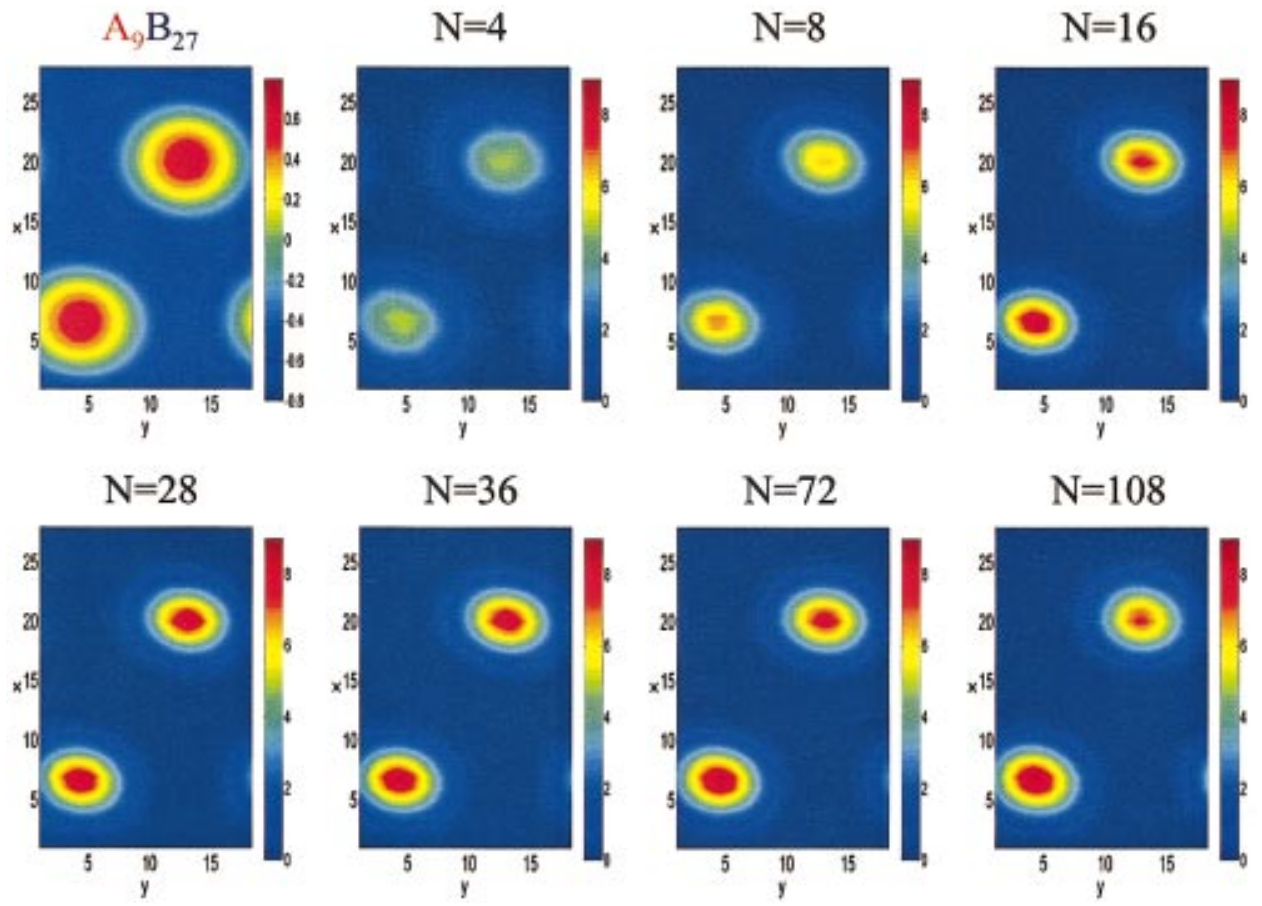

FIG. 4. (Color) Segmental distribution of the A-like homopolymer $\mathrm{H}^{\mathrm{A}}$ of length $N$ in the cylindrical structure of diblock copolymers. The first graph shows the two-dimensional order parameter profile of the copolymers in a unit cell of $27 \times 17 \frac{1}{3}$ of the hexagonal packing of the cylinders, where the color code of 0.7 corresponds to the A-rich domain (cylinders) and -0.7 corresponds to the B-rich domain (matrix). The rest shows the homopolymer segmental distribution, where the color code of 1 corresponds to the average value (the uniform distribution of the homopolymer segments in the system).
The results for neutral homopolymers $\mathrm{H}^{\mathrm{N}}$, shown in Fig. 6 , reveal a number of interesting features. For short chain lengths, $\mathrm{H}^{\mathrm{N}}$ exhibits the same behavior as neutral good solvent molecules (which correspond to $N=1$ ), i.e., it segregates at $\mathrm{A}-\mathrm{B}$ interfaces to screen the repulsion between $\mathrm{A}$ and $\mathrm{B}$ segments. For longer chain lengths, however, $\mathrm{H}^{\mathrm{N}}$ exhibits an apparent preference for the cylinders over the matrix. That is, at the same radial distance away from an A-B interface (denoted by $\left.r_{0}\right), D(x, y ; N)$ in the cylinder is larger than that in the matrix. This can be explained on the basis of geometrical considerations. Consider an A-cylinder and its surrounding B-matrix; the neutral homopolymer segments do not have an energetic preference for the A or B domain. However, the (differential) volume at a distance $r$ from the center of the cylinder is proportional to $r$; this leads to a higher density of homopolymer segments in the cylinder than that in the matrix (at the same $r_{0}$ ). Accordingly, for a lamellar morphology, this preference of the neutral homopolymers for one domain over the other disappears; in contrast, for a spherical morphology we anticipate an even stronger preference for the spheres over the matrix, because the (differential) volume at a distance $r$ from the center of a sphere is proportional to $r^{2}$.

The chain conformation of the long neutral homopoly-
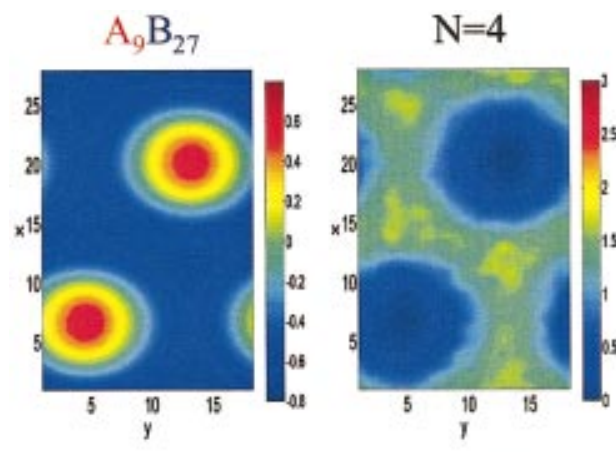

$\mathrm{N}=28$

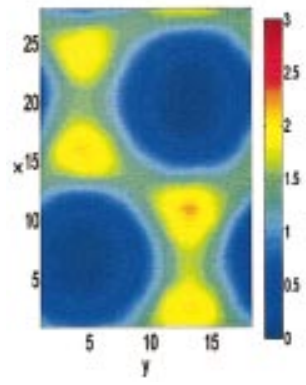

$\mathrm{N}=36$

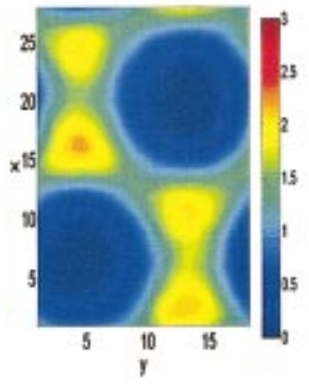

$\mathrm{N}=8$

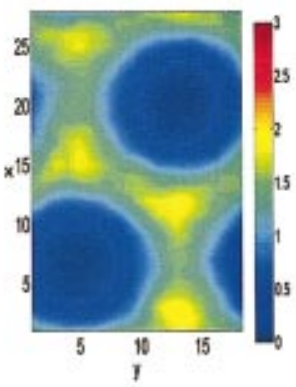

$\mathrm{N}=72$

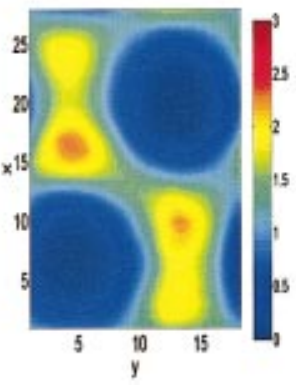

$\mathrm{N}=16$

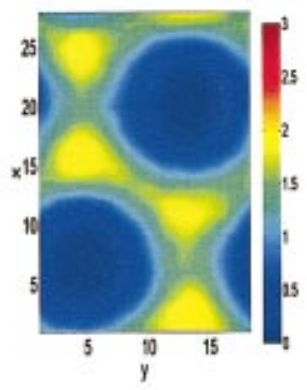

$\mathrm{N}=108$

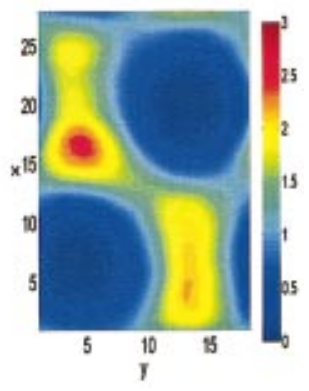

FIG. 5. (Color) Segmental distribution of the B-like homopolymer $\mathrm{H}^{\mathrm{B}+}$ of length $N$ in the cylindrical structure of diblock copolymers. Refer to Fig. 4 for more explanation. 

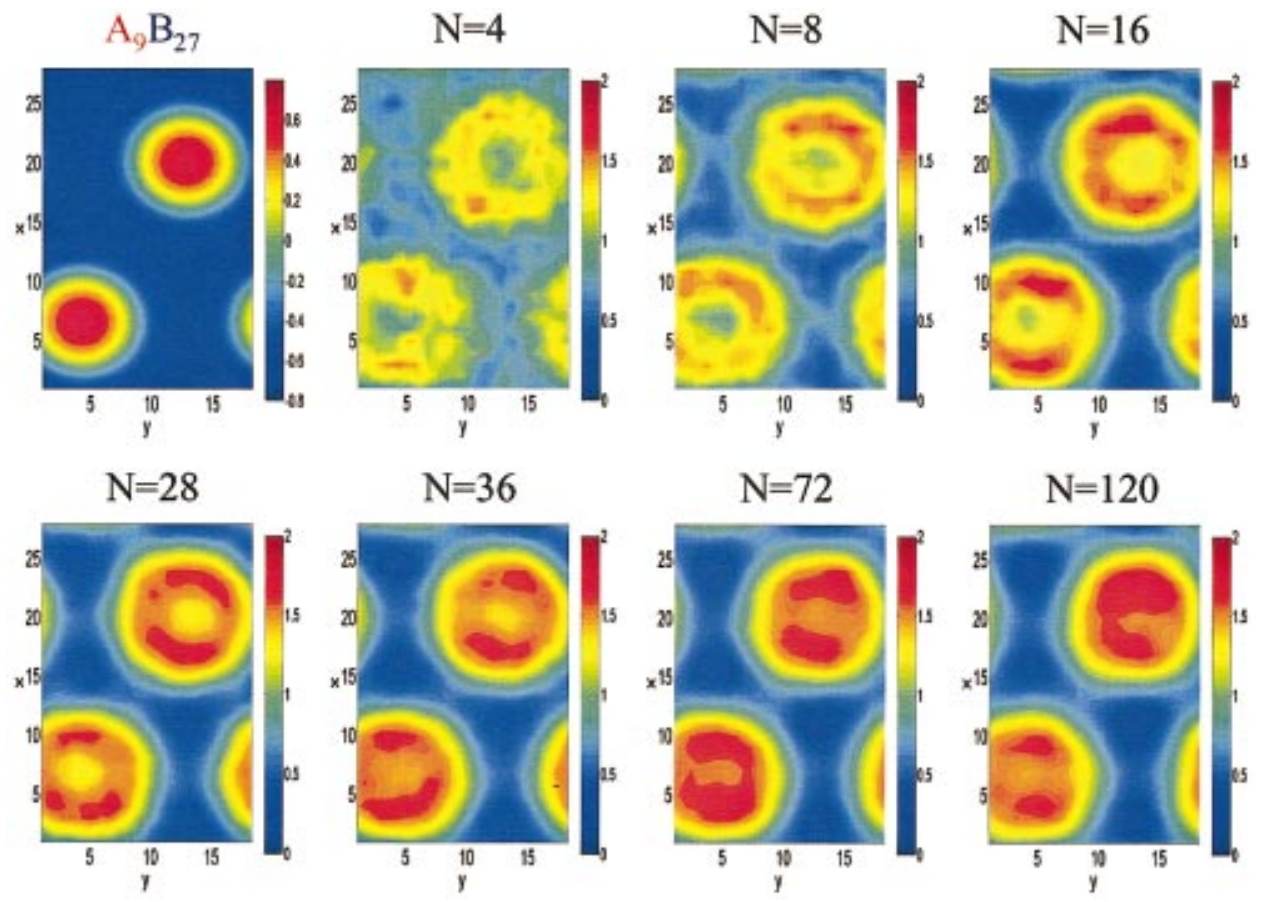

FIG. 6. (Color) Segmental distribution of the neutral homopolymer $\mathrm{H}^{\mathrm{N}}$ of length $N$ in the cylindrical structure of diblock copolymers. Refer to Fig. 4 for more explanation. mer could also influence its segmental distribution. To quantify the homopolymer chain conformation, we calculate the components of the mean square radius of gyration of the homopolymer, denoted by $R_{g, i}^{2}(i=x y, z)$, where $R_{g, x y}^{2}$ $\equiv\left(R_{g, x}^{2}+R_{g, y}^{2}\right) / 2$; the results are shown in Fig. 7 , where for each set of data a linear fit for $\ln R_{g, i}^{2} \sim \ln N$ (with $N \geqslant 16$ ) is shown and the slope is given in the legend. We see that $R_{g, z}^{2}>R_{g, x y}^{2}$ in all cases, and $R_{g, z}^{2}-R_{g, x y}^{2}$ becomes larger for longer chains. The single homopolymer chain is therefore elongated along the $z$ direction. This elongation is most pronounced for long chains of $\mathrm{H}^{\mathrm{A}}$, whose conformation resembles more a rod than a Gaussian coil. The difference between the $z$ and the other two directions is caused by the orientation of the cylinders.

We also calculate the single chain structure factor of the homopolymer,

$$
\begin{aligned}
S(\mathbf{q}) & \equiv \frac{1}{N} \sum_{k, m}^{N}\left\langle e^{j \mathbf{q} \cdot\left(\mathbf{r}_{k}-\mathbf{r}_{m}\right)}\right\rangle_{N} \\
& =\frac{1}{N}\left\langle\left[\sum_{k}^{N} \cos \left(\mathbf{q} \cdot \mathbf{r}_{k}\right)\right]^{2}+\left[\sum_{k}^{N} \sin \left(\mathbf{q} \cdot \mathbf{r}_{k}\right)\right]^{2}\right\rangle_{N},
\end{aligned}
$$

where $j=\sqrt{-1} ; \mathbf{q}$ is the wave vector; $\mathbf{r}_{k}$, for example, is the lattice position of the $k$ th segment of the homopolymer; and \langle\rangle$_{N}$ here denotes an average over collected samples having a homopolymer of length $N$. Figure 8 shows $S_{i}(q) / N(i$ $=x y, z)$, where $S_{x y}(q)$, for example, is obtained by averaging $S(\mathbf{q})$ over vectors $\mathbf{q}$ that are limited in the $x-y$ plane and have a length of $q ; S_{i}(q)$ is determined in the range $2 \pi / \max \left\{L_{x}, L_{y}, L_{z}\right\} \leqslant q \leqslant 2 \pi$. Lattice features become apparent in Fig. 8 for $q \geq 2(\ln q \geq 0.7)$.

The conformation of the single homopolymer chain can also be characterized using the scaling exponent $\nu$, defined by $S_{i}(q) \propto q^{-1 / \nu} ; \nu=0.5$ corresponds to the chain conforma-
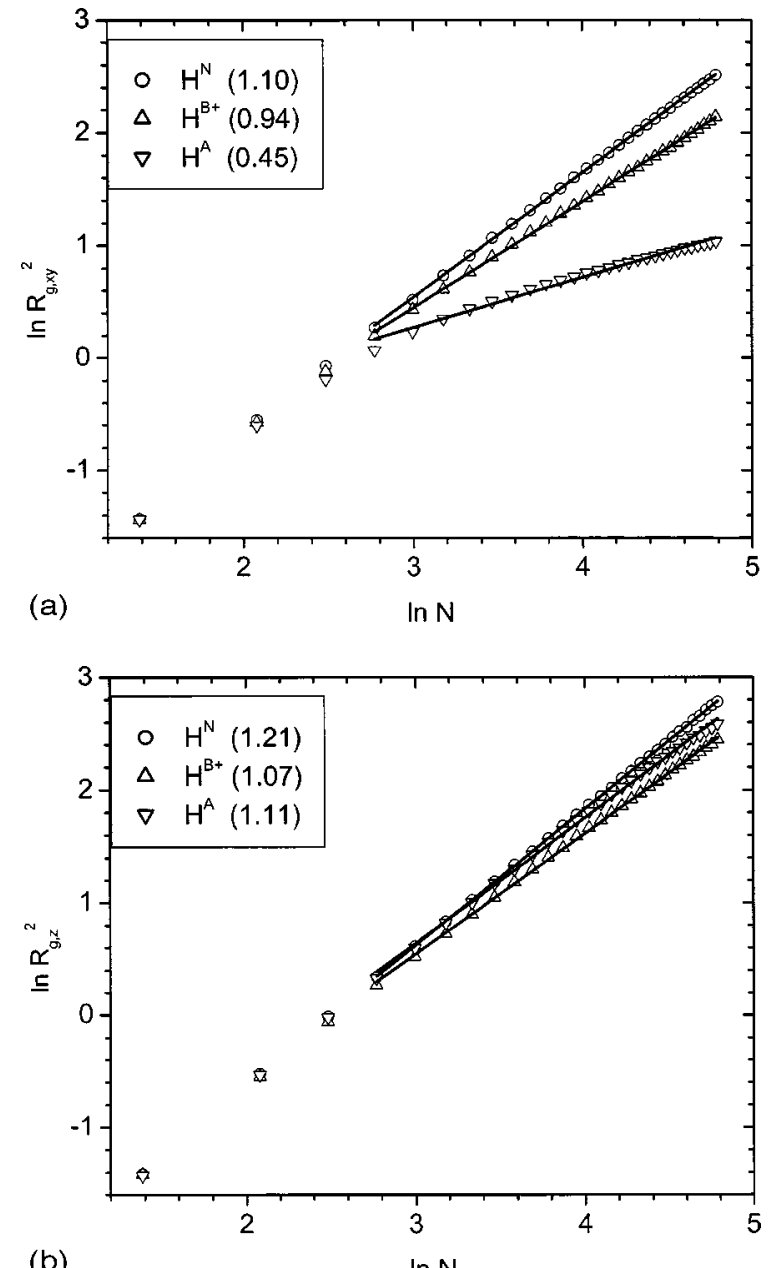

FIG. 7. The components of the mean square radius of gyration of the homopolymer $R_{g, i}^{2}(i=x y, z)$, where $R_{g, x y}^{2} \equiv\left(R_{g, x}^{2}+R_{g, y}^{2}\right) / 2$. The symbols are simulation results, with different shapes corresponding to different homopolymer interactions as shown in the legend. Each line represents the linear fit for the corresponding set of data (where only the results for $N$ $\geqslant 16$ are used), with its slope given in the legend. 

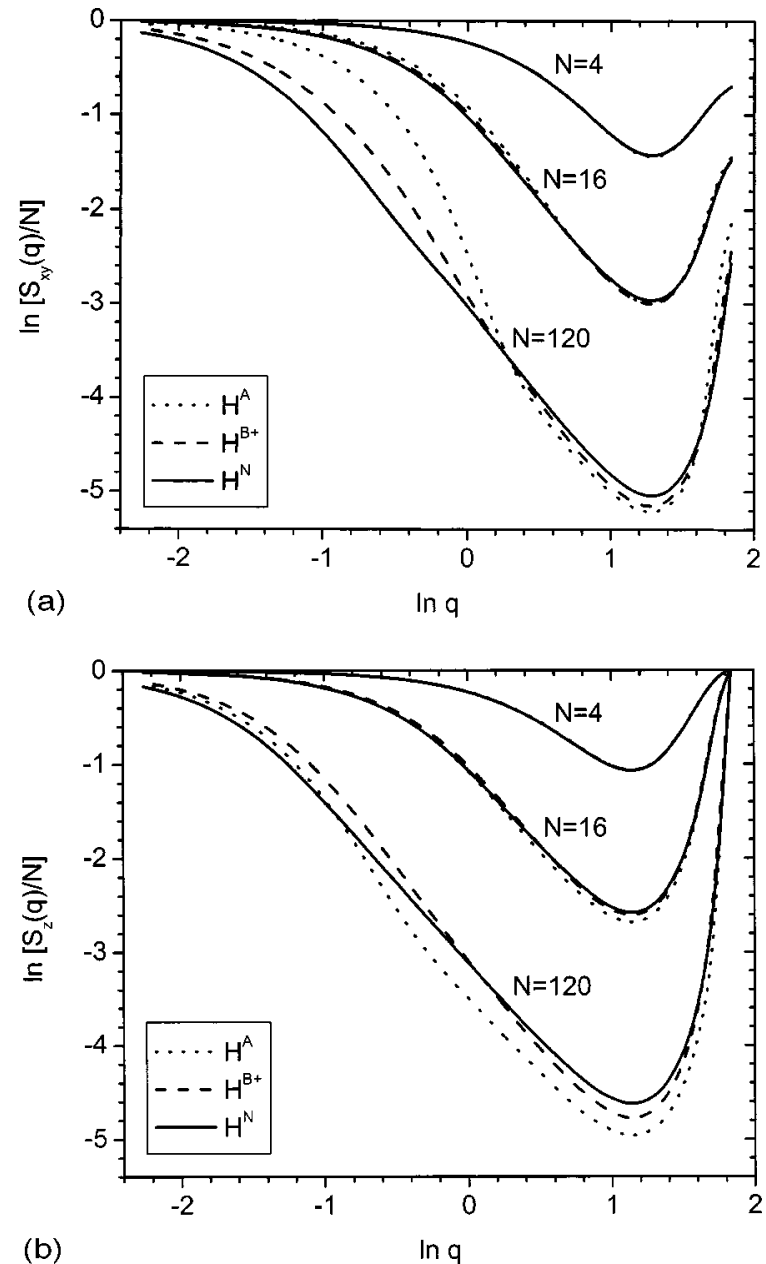

FIG. 8. The single chain structure factor $S_{i}(q)(i=x y, z)$ of the homopolymer of length $N$.

tion in a $\theta$ solvent, $\nu<0.5$ corresponds to a compressed conformation in a poor solvent, and $\nu>0.5$ corresponds to an extended conformation in a good solvent. Figure 9 shows the value of $\nu$ for the longest homopolymer chain of $N=120$, obtained from Fig. 8. For $q \leqslant 0.45(\ln q \leqslant-0.8), \nu$ monotonically decreases as $q$ increases in all cases. It is interesting to see that, for the A-like homopolymer $\mathrm{H}^{\mathrm{A}}, S_{z}(q)$ exhibits two regimes (at $q$ around 0.5 and 1.5) with different values of $\nu$, while $S_{x y}(q)$ hardly exhibits a regime of constant $\nu$. All other curves in Fig. 9 exhibit one regime with an almost constant $\nu$, which compares favorably with the corresponding slope of the linear fit for $\ln R_{g, i}^{2} \sim \ln N$ shown in Fig. 7. Note that, for the $\mathrm{H}^{\mathrm{A}}$, the slope of $\ln R_{g, z}^{2} \sim \ln N$ is close to the average value of $2 \nu$ in the two different regimes, while the $\ln R_{g, x y}^{2}$ $\sim \ln N$ data cannot be well-fitted by a linear function.

\section{CONCLUSIONS}

We have performed Monte Carlo simulations to study the behavior of a single nanoparticle in lamellae of symmetric diblock copolymers. The particle is fixed at the center of the simulation box, and the lamellae move during our simulations performed in an expanded grand-canonical ensemble. We find that "small" particles (with their size smaller than half the lamellar period $L$ ) can be located in the preferred
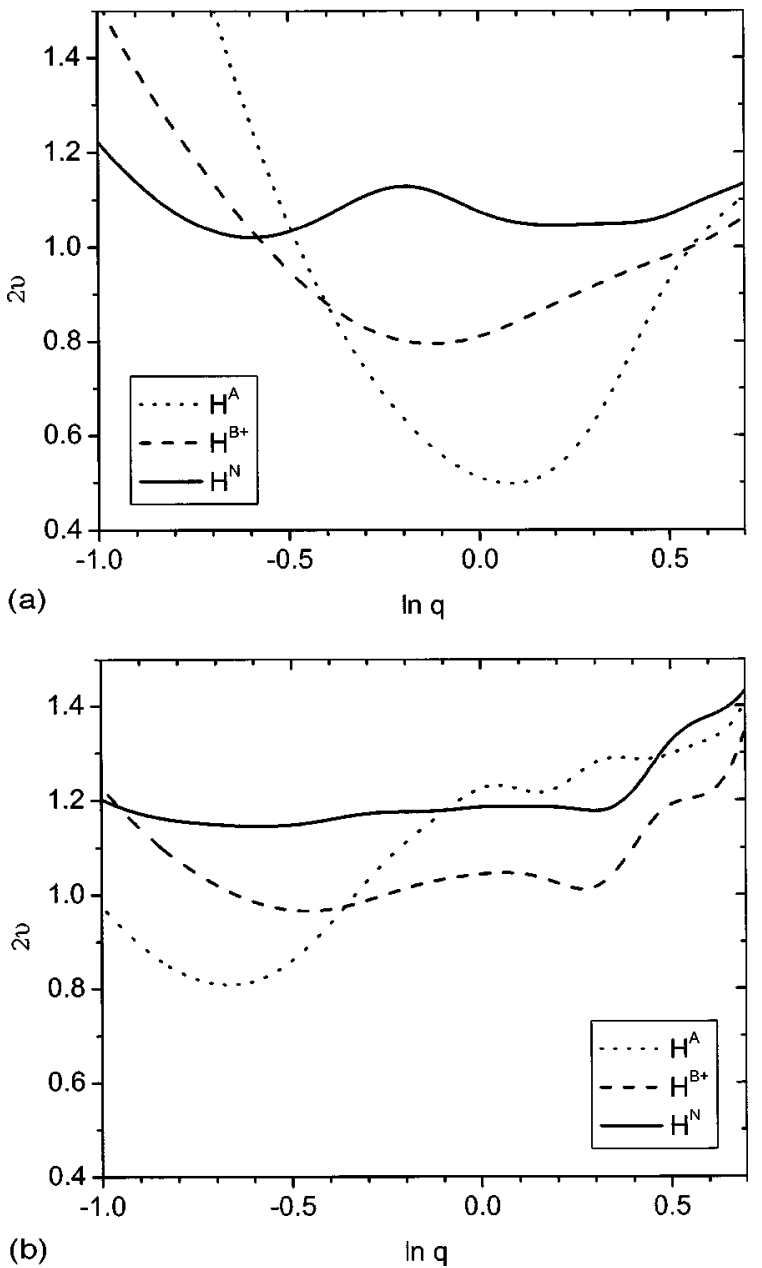

FIG. 9. The scaling exponent $\nu$ for the homopolymer of $N=120$, determined from (a) $S_{x y}(q)$ and (b) $S_{z}(q)$ shown in Fig. 8, respectively.

lamellar domain according to their interactions with the blocks (at the A-B interfaces if they are neutral), and the lamellar structure is not disturbed by the particle. In contrast, "large" particles (with their size larger than $L / 2$ ) disturb the lamellar structure if they are not neutral, due to the surrounding of the preferred segments around the particle. For the particle sizes considered here, the shape of the particle (cubic or spherical) does not have a significant influence on its behavior.

We have also reported Monte Carlo simulations of a single homopolymer chain in cylinders of asymmetric diblock copolymers. The chain length of the homopolymer is varied using expanded grand-canonical (growing/shrinking) moves. We find that homopolymers preferring the shorter blocks are highly localized in the cylinders, consistent with recent experimental observations. ${ }^{14}$ We also find that this localization becomes stronger for longer chains. While short neutral homopolymers segregate at the $\mathrm{A}-\mathrm{B}$ interfaces to screen the repulsion between A and B segments, longer neutral homopolymers exhibit an apparent preference for the cylinders over the matrix, mainly due to geometrical considerations associated with the curvature of A-B interfaces. 


\section{ACKNOWLEDGMENTS}

The authors are grateful to Dr. Jin Kon Kim and Dr. Thomas Russell for bringing the problem of dilute homopolymers in cylinders of diblock copolymers to our attention, and for helpful discussions on this problem. Financial support for this work was provided by the Semiconductor Research Corporation through Contract No. 99-LP-452 and NSF CTS-0210588.

\footnotetext{
${ }^{1}$ See, for example, (a) M. Park, C. Harrison, P. M. Chaikin, R. A. Register, and D. H. Adamson, Science 276, 1401 (1997); (b) T. Hashimoto, K. Tsutsumi, and Y. Funaki, Langmuir 13, 6869 (1997); (c) G. Liu, J. Ding, T. Hashimoto, K. Kimishima, F. M. Winnik, and S. Nigam, Chem. Mater. 11, 2233 (1999); (d) R. G. H. Lammertink, M. A. Hempenius, J. E. van den Enk, V. Z.-H. Chan, E. L. Thomas, and G. J. Vancso, Adv. Mater. 12, 98 (2000); (e) R. D. Peters, X. M. Yang, Q. Wang, J. J. de Pablo, and P. F. Nealey, J. Vac. Sci. Technol. B 18, 3530 (2000); (f) T. Thurn-Albrecht, J. Schotter, G. A. Kastle, N. Emley, T. Shibauchi, L. Krusin-Elbaum, K. Guarini, C. T. Black, M. T. Tuominen, and T. P. Russell, Science 290, $2126(2000)$.

${ }^{2}$ See, for example, (a) R. E. Cohen, Curr. Opin. Solid State Mater. Sci. 4, 587 (1999); (b) R. W. Zehner and L. R. Sita, Langmuir 15, 6139 (1999); (c) Y. Boontongkong, R. E. Cohen, and M. F. Rubner, Chem. Mater. 12, 1628 (2000); (d) W. A. Lopes and H. M. Jaeger, Nature (London) 414, 735 (2001).

${ }^{3}$ See, for example, (a) M. Templin, A. Franck, A. Du Chesne, H. Leist, Y. Zhang, R. Ulrich, V. Schadler, and U. Wiesner, Science 278, 1795 (1997); (b) D. Zhao, J. Feng, Q. Huo, N. Melosh, G. H. Fredrickson, B. F. Chmelka, and G. D. Stucky, ibid. 279, 445 (1998); (c) V. Z.-H. Chan, J.
}

Hoffman, V. Y. Lee, H. Iatrou, A. Avgeropoulos, N. Hadjichristidis, R. D. Miller, and E. L. Thomas, ibid. 286, 1716 (1999).

${ }^{4}$ G. J. A. Sevink, A. V. Zvelindovsky, B. A. C. van Vlimmeren, N. M. Maurits, and J. G. E. M. Fraaije, J. Chem. Phys. 110, 2250 (1999).

${ }^{5}$ V. V. Ginzburg, C. Gibbons, F. Qiu, G. Peng, and A. C. Balazs, Macromolecules 33, 6140 (2000).

${ }^{6}$ V. V. Ginzburg, F. Qiu, and A. C. Balazs, Polymer 43, 461 (2002).

${ }^{7}$ J. Huh, V. V. Ginzburg, and A. C. Balazs, Macromolecules 33, 8085 (2000).

${ }^{8}$ R. B. Thompson, V. V. Ginzburg, M. W. Matsen, and A. C. Balazs, Science 292, 2469 (2001).

${ }^{9}$ R. B. Thompson, V. V. Ginzburg, M. W. Matsen, and A. C. Balazs, Macromolecules 35, 1060 (2002).

${ }^{10}$ J. Y. Lee, R. B. Thompson, D. Jasnow, and A. C. Balazs, Macromolecules 35, 4855 (2002).

${ }^{11}$ Q. Wang, Q. Yan, P. F. Nealey, and J. J. de Pablo, J. Chem. Phys. 112, 450 (2000).

${ }^{12}$ Q. Wang, P. F. Nealey, and J. J. de Pablo, Macromolecules 34, 3458 (2001).

${ }^{13}$ See, for example, K. Binder and M. Muller, Curr. Opin. Colloid Interface Sci. 5, 315 (2000), and references therein.

${ }^{14}$ U. Jeong, H.-C. Kim, R. L. Rodriguez, I. Y. Tsai, C. M. Stafford, J. K. Kim, C. J. Hawker, and T. P. Russell, Adv. Mater. 14, 274 (2002).

${ }^{15}$ Q. Wang, P. F. Nealey, and J. J. de Pablo, Macromolecules 35, 9563 (2002).

${ }^{16}$ Due to the simulation box size, the cylinders are slightly stretched in the $y$ direction and are slightly compressed in the $x$ direction. This deformation is small (about 3\% of our estimated bulk dimensions of cylinders in Ref. 12 ), and does not affect our results qualitatively.

${ }^{17}$ M. Kikuchi and K. Binder, J. Chem. Phys. 101, 3367 (1994).

${ }^{18}$ Q. Wang, P. F. Nealey, and J. J. de Pablo, Macromolecules 36, 1731 (2003) 